\title{
Clinical case report: conservative treatment of nodular adenomyosis
}

\begin{abstract}
This article describes a clinical case report of nodular adenomyosis first diagnosed in adolescent girl. It emphasizes the importance of early disease detection and correct choice of therapeutic approach factors, directly relating to the future reproductive health of an adolescent girl. This case describes an example of successful long-term conservative treatment of adenomyosis with dienogest (Visanne) treatment for over three years and subsequent realization of reproductive function by the patient
\end{abstract}

Keywords: nodular adenomyosis, adolescence, visanne, deterioration, problem, endometriosis
Volume 8 Issue 2 - 2019

\author{
Zakharenko NF, Kovalenko NV, Manoliak IP \\ Department of Gynecology, Kyiv Municipal Hospital No. 9, \\ Ukraine
}

\begin{abstract}
Correspondence: Zakharenko NF, Department of Gynecology, Kyiv Municipal Hospital No. 9, Ukraine, Tel +380 (44) 455695, Emailmajid.eslami@modares.ac.ir
\end{abstract}

Received: March 15, 2018| Published: April 02, 2019

\section{Introduction}

These days, the problem of endometriosis has become ever more relevant. ${ }^{1-3}$ Environmental deterioration, increased emotional stress load in the society contribute to the formation of tension in the neuro humoral regulatory systems predisposing to the emergence of various hyper proliferative pathologies, including endometriosis. ${ }^{4-6}$

The problem of endometriosis has long ceased to be purely medical because it is associated with reduced quality of life of the women of reproductive age - the most socially important category. ${ }^{5,7}$ Currently, endometriosis is no longer associated with reproductive age only as there is an increasing incidence of this condition being diagnosed in adolescence and at that time it accounts for $70 \%$ cases of chronic pelvic pain. ${ }^{2,3}$ Numerous studies have proven that endometriosis may occur in adolescents girls., ${ }^{2,8,7}$ The average age of disease onset is 15.9 years. Therefore, timely diagnosis of endometriosis and choice of adequate therapeutic approach are of particular importance in this patient population since they predetermine the possibility of successful reproductive performance of such women in the future. ${ }^{2,7,9}$

Having regard to the above, the case history of adenomyosis first diagnosed in adolescence deserves close attention. On June 6,2013 , patient M. aged 18 was admitted on an urgent basis to the gynecology department of Kyiv Municipal Hospital No.9. The patient presented with severe pain during menstruation, low-grade fever, nausea, vomiting. Referral diagnosis: nodular uterine leiomyoma with impaired nodular delivery. From the medical history of the patient: menstruation first started at the age of 13 and regular cycle was established within a year, periods were painful, sometimes leading to fainting. The patient also experienced loosed stool during the first days of periods. The patient repeatedly applied to pediatric gynecology department where she was diagnosed with the following: Dysmenorrhea. Adenomyosis? In 2011, she was examined by the gynecologist of the "Okhmatdyt" National Children's Specialized Hospital with subsequent pelvic ultrasound examination. Diagnosis: Nodular adenomyosis $(72 \times 68 \mathrm{~mm}$; endometrial nodule was identified on the posterior uterine wall in the myometrium). The patient was prescribed 6 injections of Diphereline $3.62 \mathrm{mg}$. The reduction in the size of nodule to $31 \times 29 \mathrm{~mm}$ has been observed under the course of the treatment. Diphereline was discontinued due to undesirable effects such as bone pain, hot flushes, fatigue, and tachycardia. Following withdrawal of Diphereline, the periods resumed in 3 months and in 6 months menstrual pain developed again.

The data of patient's physical examination: the skin and visible mucous membranes are of pale pink color, the abdomen is soft, tender on palpation, participates in the process of breathing. Peritoneal signs are weakly positive in the lower abdomen. Pasternatsky's symptom is negative on both sides. Vaginal examination: uterus is enlarged as with 8-9 weeks pregnancy, round-shaped, dense, tender on palpation, cervical tractions are painless. Uterine appendages on both sides are not enlarged and painless on palpation. Ultrasound examination: uterus $69 \times 64 \times 62 \mathrm{~mm}$. A hypoechoic mass is visible in the structure of myometrium on the posterior wall with hyperechoic dispersed particulate matter $50 \times 46 \times 43 \mathrm{~mm}$ (Figure 1).

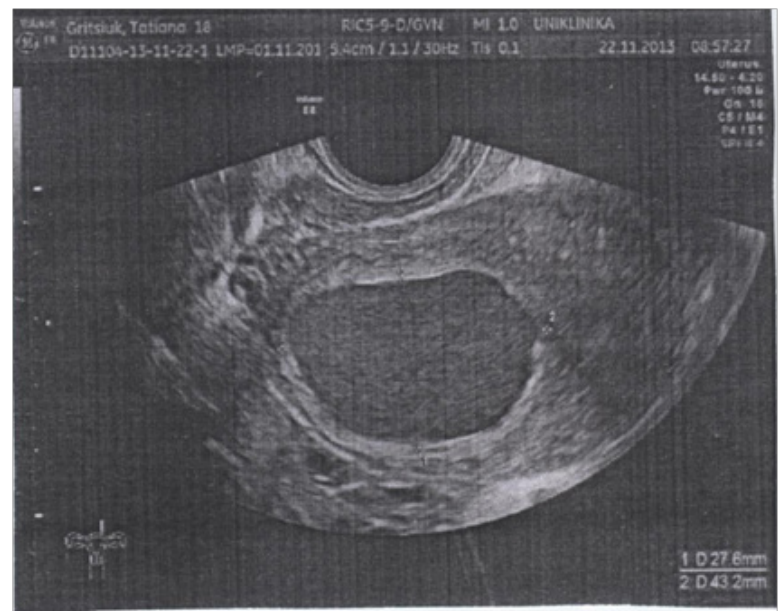

Figure I Patient M. Nodular adenomyosis (June 20I3).

Diagnosis: adenomyosis, nodular form. Secondary dysmenorrhea. Continuous treatment with Visanne over the period of 9 months has been recommended. The patient was examined in 9 months, in March 2014. The general state of health was assessed by the patient as satisfactory. Pain disappeared in as little as 3 months of treatment with Visanne. Findings of pelvic ultrasound: uterus 56x52x48mm. A hypoechoic mass is present in the structure of myometrium on the posterior wall with hyperechoic particulate matter $41 \times 36 \times 28 \mathrm{~mm}$ (Figure 2). 


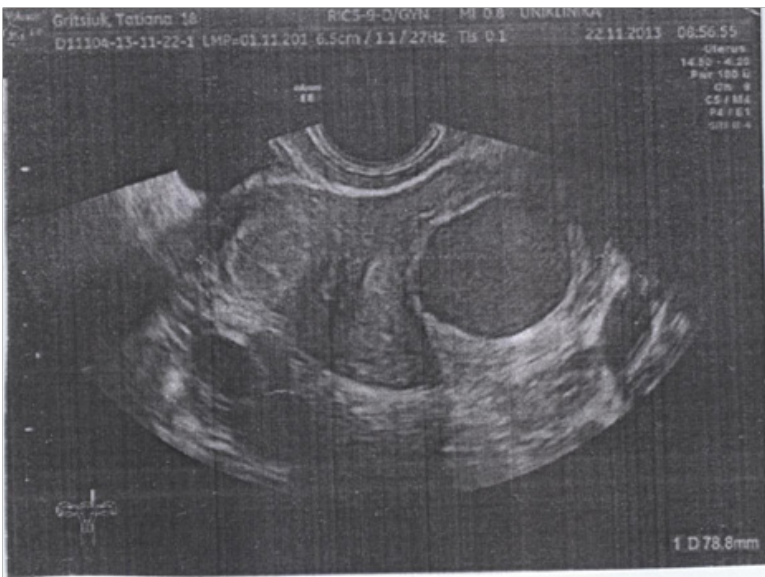

Figure 2 Patient M. Nodular adenomyosis (March 2014).

Recommended: continued treatment with Visanne for one year.

In March 2015, the patient visited the clinic for follow-up examination. According to the patient, she decided to discontinue treatment with Visanne three months ago due to the prolonged complaint-free period. At the time of examination, the patient reported reoccurrence of painful menstruation in February 2015. The pelvic ultrasound has been performed. Findings: uterus $53 \times 42.6 \times 44 \mathrm{~mm}$. A hypoechoic mass is present in the structure of myometrium on the

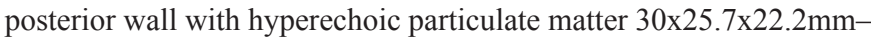
endometrioma. The patient was recommended to continue treatment with Visanne.

In January 2016 (in 11 months), the patient made follow-up visit. The general state of health was assessed by the patient as good. Findings of pelvic ultrasound: uterus $44 \times 38 \times 36 \mathrm{~mm}$. A hypoechoic mass is present in the structure of myometrium on the posterior wall with hyperechoic particulate matter $21 \times 16.7 \times 16.2 \mathrm{~mm}$ (Figure 3 ). The patient was recommended to continue treatment with Visanne. In April 2017, as the patient got married and started unprotected sexual life, Visanne was discontinued and replaced by Epigalin (composition: $200 \mathrm{mg}$ indol-3-carbinol-200, $82 \mathrm{mg}$ green tea extract). There were no complaints expressed by the patient at the time of examination. Findings of ultrasound: uterus $46 \times 39 \times 38 \mathrm{~mm}$. A hypoecho mass is presented in the structure of myometrium with hyperechoic particulate matter $29.0 \times 20.7 \times 18.9 \mathrm{~mm}$. The patient was recommended to continue treatment with Epigalin. In December 2017, the patient sought advice of gynecologist because of 14-days delay of menstruation. Ultrasound examination: uterus $56.2 \times 50.8 \times 49.4 \mathrm{~mm}$. Gestational sac $16.6 \times 16.1 \mathrm{~mm}$ is visible in the uterine cavity. A hypoechoic mass is visible in the myometrium with hyperechoic particulate matter $18.1 \mathrm{x}$ $11.1 \mathrm{~mm}$ (Figure 4).

\section{Physician's statement: pregnancy 4 weeks. Nodular adenomyosis}

The patient made scheduled visit on 02.02.2018. The general state of health was satisfactory. Normal course of pregnancy. Ultrasound findings: uterine body: 139.0x74.0x103.0 mm; 113.0x44.0x57.0 mm gestational sac is visible in the uterine cavity; cervix: $37.0 \mathrm{~mm}$. Internal orifice of the uterus is closed; uterine walls: no abnormalities; uterine tonus: no abnormalities. Cervical canal: no abnormalities. Ultrasound report: Pregnancy 13 weeks+05 days Progressing (Figure 5).

On 09.03.2018, the patient underwent the second scheduled ultrasound. Conclusion: Pregnancy 18 weeks. Progressing (Figure 6).

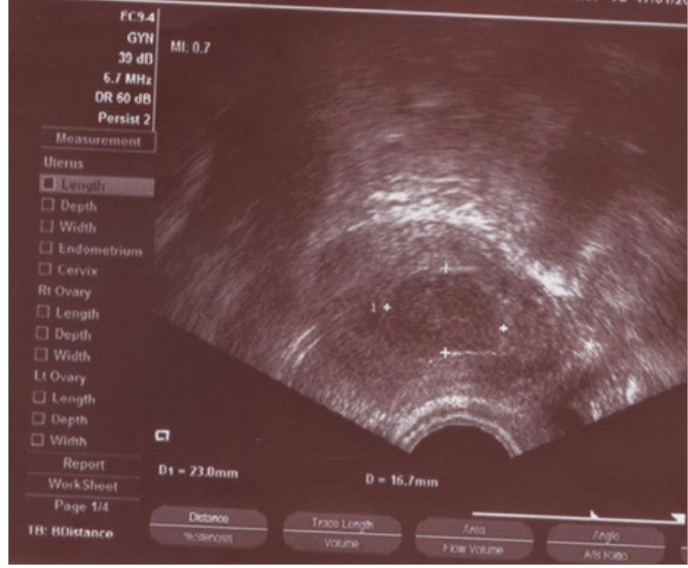

Figure 3 Patient M. Nodular adenomyosis (January 20I6).

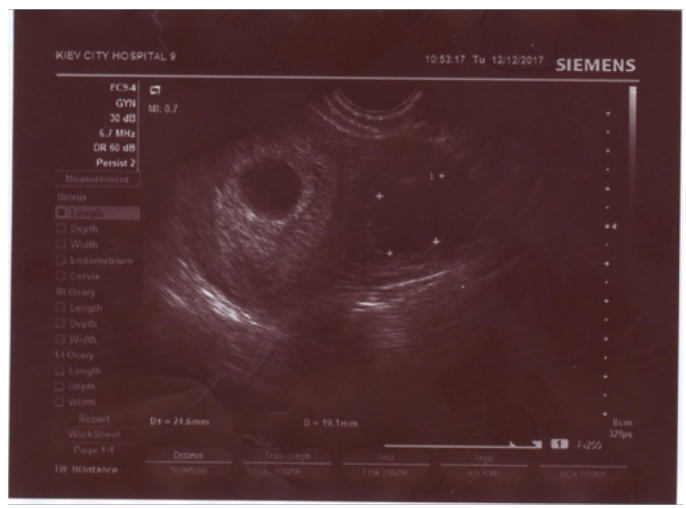

Figure 4 Patient M. Pregnancy 4 weeks. Nodular adenomyosis (December 2017).

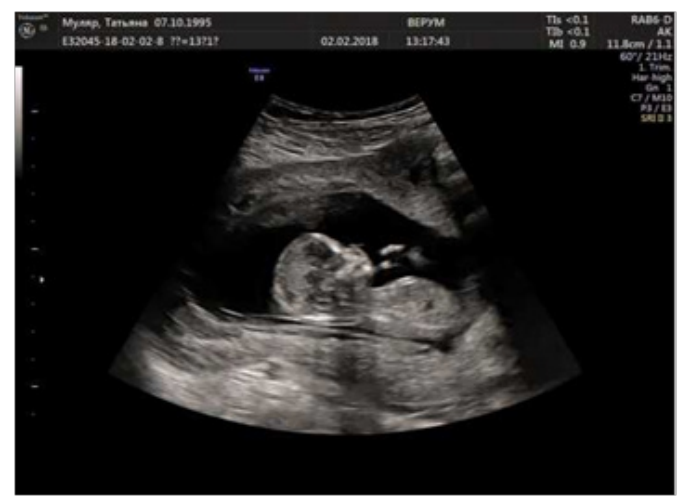

Figure 5 Patient M. Pregnancy I 3 weeks Progressing.

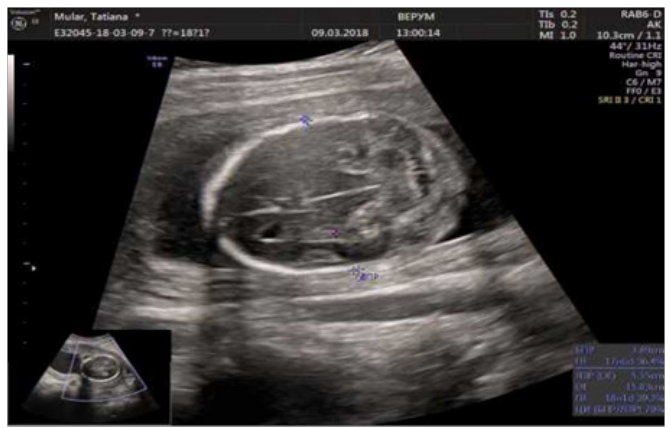

Figure 6 Patient M. Follow-up ultrasound examinations at pregnancy week 18. 


\section{Conclusion}

Secondary dysmenorrhea with underlying endometriosis is a highly challenging issue in modern gynaecology. Unfortunately, statistical data demonstrate that this condition is often diagnosed in 6-7 years after the appearance of complaints. Considering the fact that endometriosis is diagnosed in $30 \%-40 \%$ of patients with primary infertility, the timely disease detection and correct choice of therapeutic approach are of vital importance since they are directly related to the future reproductive health of an adolescent girl. The nodular adenomyosis, even with large nodules, can be successfully treated conservatively with dienogest, and such long-term therapy (for over three years) is well-tolerated by the patients and contributes to the effective implementation of their reproductive function.

\section{Acknowledgments}

None.

\section{Conflicts of interest}

The author declares there no conflict of interest here.

\section{References}

1. Tatarchuk TF, Kalugina LV. On the issue of prevention and treatment of hormone-dependent hyper proliferative diseases in women. Women's Health. 2013;7(1):51-57.
2. Guideline of the European Society of Human Reproduction and Embryology. Management of endometriosis. 2013.97 p.

3. Dunselman GA, Vermeulen N, Becker C, et al. ESHRE guideline: management of women with endometriosis. Hum Reprod. 2014;29(3):400-412.

4. Vovk IB, Zakharenko NF, Radysh TV. Status of activation of blood lymphocytes and serum levels of inflammatory mediators in different forms of endometriosis. Pediatrics, obstetrics and gynecology. 2013;76(1):77-81.

5. Zakharenko NF, Zadorozhnaia TD, Kalugina LV. Indol-3-carbinol in the treatment of adenomyosis. F1000Res. 2013;2(15):22-24.

6. Zakharenko NF, Tatarchuk TF, Kovalenko NV. The role of oxidative stress in the genesis of endometriosis. Reproductive endocrinology. 2014;4(18):13-16.

7. Stilley JA. Cellular and molecular basis for endometriosis-associated infertility. Cell Tissue Res. 2012;349(3):849-862.

8. Johnson NP. Consensus on current management of endometriosis. Hum Reprod. 2013;28(6):1552-1568.

9. Zotova OA, Artymuk NV. Adenomyosis: clinic, risk factors and problems of diagnosis and treatment. Gynecology. 2013;5(6):31-34. 